\title{
Front Matter: Volume 6486
}

, "Front Matter: Volume 6486," Proc. SPIE 6486, Light-Emitting Diodes:

Research, Manufacturing, and Applications XI, 648601 (8 March 2007); doi: 10.1117/12.727556

SPIE Event: Integrated Optoelectronic Devices 2007, 2007, San Jose, California, SPIE. United States 


\title{
PROCEEDINGS OF SPIE
}

\section{Light-Emitting Diodes: Research, Manufacturing, and Applications XI}

\author{
Klaus P. Streubel \\ Heonsu Jeon \\ Editors
}

24-25 January 2007

San Jose, Calfornia, USA

Sponsored by

SPIE-The International Society for Optical Engineering

Optical Research Associates (USA)

Published by

SPIE-The International Society for Optical Engineering

Volume 6486

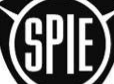

The International Society for Optical Engineering

Proceedings of SPIE-The International Society for Optical Engineering, 9780819465993, v. 6486

SPIE is an international technical society dedicated to advancing engineering and scientific applications of optical, photonic, imaging, electronic, and optoelectronic technologies. 
The papers included in this volume were part of the technical conference cited on the cover and title page. Papers were selected and subject to review by the editors and conference program committee. Some conference presentations may not be available for publication. The papers published in these proceedings reflect the work and thoughts of the authors and are published herein as submitted. The publisher is not responsible for the validity of the information or for any outcomes resulting from reliance thereon.

Please use the following format to cite material from this book:

Author(s), "Title of Paper," in Light-Emitting Diodes: Research, Manufacturing, and Applications XI, edited by Klaus P. Streubel, Heonsu Jeon, Proceedings of SPIE Vol. 6486 (SPIE, Bellingham, WA, 2007) Article CID Number.

ISSN 0277-786X

ISBN 9780819465993

Published by

SPIE-The International Society for Optical Engineering

P.O. Box 10, Bellingham, Washington 98227-0010 USA

Telephone 1 360/676-3290 (Pacific Time) · Fax 1 360/647-1445

http://www.spie.org

Copyright (C) 2007, The Society of Photo-Optical Instrumentation Engineers

Copying of material in this book for internal or personal use, or for the internal or personal use of specific clients, beyond the fair use provisions granted by the U.S. Copyright Law is authorized by SPIE subject to payment of copying fees. The Transactional Reporting Service base fee for this volume is $\$ 18.00$ per article (or portion thereof), which should be paid directly to the Copyright Clearance Center (CCC), 222 Rosewood Drive, Danvers, MA 01923. Payment may also be made electronically through CCC Online at http://www.copyright.com. Other copying for republication, resale, advertising or promotion, or any form of systematic or multiple reproduction of any material in this book is prohibited except with permission in writing from the publisher. The CCC fee code is 0277 $786 \times / 07 / \$ 18.00$

Printed in the United States of America. 


\section{Contents}

vii Conference Committee

NOVEL TECHNOLOGIES FOR LEDS

648604 Fundamental mechanisms of electroluminescence refrigeration in heterostructure lightemitting diodes [6486-03]

S.-Q YU, J.-B. Wang, D. Ding, S. R. Johnson, D. Vasileska, Y.-H. Zhang, Arizona State Univ. (USA)

648605 Nonperiodic binary optical structures for enhancing light extraction of emitters [6486-04]

L. Hong, X. HU, T. YU, T. Dai, Z. Zhang, J. XU, G. Zhang, Peking Univ. (China)

648606 High light-extraction efficiency in GalnN light-emitting diode with pyramid reflector [6486-05]

J.-Q. Xi, H. Luo, J. K. Kim, E. F. Schubert, Rensselaer Polytechnic Institute (USA)

648607 LED engine hosted on a THS [6486-06]

M. Checchetti, micrOptronics (Italy)

\section{FABRICATION OF LEDS AND OLEDS}

64860A Dicing of high-power white LEDs in heat sinks with the water jet-guided laser [6486-09]

T. A. Mai, R. Housh, A. Brulé, B. Richerzhagen, Synova S.A. (Switzerland)

64860C Integration of high-efficiency PIN organic light-emitting devices in lighting and

optoelectronic applications (Invited Paper) [6486-1 1]

J. Amelung, M. Toerker, Y. Tomita, D. Kreye, C. Grillberger, U. Vogel, A. Elgner, M. Eritt, Ch. May, U. Todt, C. Luber, R. Hermann, Ch. Zschippang, Fraunhofer Institute for Photonic Microsystems (Germany); K. Leo, Fraunhofer Institute for Photonic Microsystems (Germany) and Technical Univ. Dresden (Germany)

Pagination: Proceedings of SPIE follow an e-First publication model, with papers published first online and then in print and on CD-ROM. Papers are published as they are submitted and meet publication criteria. A unique, consistent, permanent citation identifier (CID) number is assigned to each article at the time of the first publication. Utilization of CIDs allows articles to be fully citable as soon they are published online, and connects the same identifier to all online, print, and electronic versions of the publication.

SPIE uses a six-digit CID article numbering system in which:

- The first four digits correspond to the SPIE volume number.

- The last two digits indicate publication order within the volume using a Base 36 numbering system employing both numerals and letters. These two-number sets start with 00, 01, 02, 03, 04, 05, 06, 07, 08, 09, 0A, 0B ... 0Z, followed by 10-1Z, 20-2Z, etc.

The CID number appears on each page of the manuscript. The complete citation is used on the first page, and an abbreviated version on subsequent pages. 
64860D New architectures for high-performance polymer light-emitting diodes introducing a solution-processed titanium oxide layer (Invited Paper) [6486-12]

S. H. Kim, J. Y. Kim, S. H. Park, K. Lee, Pusan National Univ. (South Korea)

64860E OLED lighting: light where it never has been before (Invited Paper) [6486-13]

M. Klein, K. Heuser, F. Schindler, B. Krummacher, T. Dobbertin, Osram Opto Semiconductors GmbH (Germany); R. Pätzold, C. Gärditz, Siemens AG (Germany)

64860F See-through OLED displays (Invited Paper) [6486-14]

W. Kowalsky, P. Görrn, J. Meyer, M. Kröger, H.-H. Johannes, T. Riedl, Technische Univ. Braunschweig (Germany)

64860G Fabrication of polymer LEDs by resonant infrared pulsed laser ablation [6486-15]

S. L. Johnson, C. T. Bowie, B. Ivanov, Vanderbilt Univ. (USA); H. K. Park, AppliFlex, LLC (USA);

R. F. Haglund, Jr., Vanderbilt Univ. (USA)

$64860 \mathrm{H}$ Employing a 2D surface grating to improve light out coupling of a substrate emitting organic LED [6486-16]

P. Vandersteegen, Univ. Gent-IMEC (Belgium); A. U. Nieto, Univ. Gent (Belgium);

C. Van Buggenhout, S. Verstuyft, P. Bienstman, P. Debackere, Univ. Gent-IMEC (Belgium);

K. Neyts, Univ. Gent (Belgium); R. Baets, Univ. Gent-IMEC (Belgium)

\section{LED CHARACTERIZATION AND RELIABILITY}

64860J Measurement of the internal quantum efficiency of InGaN quantum wells [6486-18]

A. Laubsch, M. Sabathil, G. Bruederl, Osram Opto Semiconductors (Germany); J. Wagner, Fraunhofer-Institut für Angewandte Festkörperphysik (Germany); M. Strassburg, E. Baur, Osram Opto Semiconductors (Germany); H. Braun, U. T. Schwarz, Univ. of Regensburg (Germany); A. Lell, S. Lutgen, N. Linder, R. Oberschmid, B. Hahn, Osram Opto Semiconductors (Germany)

64860K Design and implementation of real-time LED spatial radiance measurement systems [6486-19]

G.-W. Chang, Z.-M Yeh, C.-C. Liao, National Taiwan Normal Univ. (Taiwan)

64860L Studies of InGaN LEDs degradation [6486-20]

O. I. Rabinovich, F. I. Manyakhin, E. K. Naimi, Moscow State Institute of Steel and Alloys, Technological Univ. (Russia); S. G. Nikiforov, ATV Outdoor Systems (Russia); V. P. Sushkov, I. G. Ermoshin, Moscow State Institute of Steel and Alloys, Technological Univ. (Russia);

A. V. Shishov, Acol Technologies SA (Russia)

64860M Prediction of intensity and color degradation of LEDs [6486-21]

M. Bürmen, F. Pernuš, B. Likar, Univ. of Ljubljana (Slovenia)

$64860 \mathrm{~N} T$ Thermally induced stresses resulting from coefficient of thermal expansion differentials between an LED sub-mount material and various mounting substrates [6486-22]

C. DeMilo, C. Bergad, R. Forni, T. Brukilacchio, Innovations in Optics, Inc. (USA) 
64860Q Manufacturing implications for photonic crystal patterning using imprint lithography (Invited Paper) [6486-25]

C. Jones, D. Lentz, G. Doyle, M. Miller, M. Ganapathisubramanian, X. LU, D. Resnick,

D. L. LaBrake, Molecular Imprints, Inc. (USA)

64860R Photonic quasi-crystal LEDs: design, modeling, and optimisation (Invited Paper) [6486-26]

M. D. B. Charlton, Univ. of Southampton (United Kingdom) and Mesophotonics Ltd. (United Kingdom); M. E. Zoorob, T. Lee, Mesophotonics Ltd. (United Kingdom)

$64860 S$ Modeling of GaN-based resonant-cavity light-emitting diode [6486-27]

Z. S. Li, Z. Q. Li, Crosslight Software, Inc. (Canada); R.-H. Horng, National Chung Hsing Univ. (Taiwan)

64860T A generalized 2D and 3D white LED device simulator integrating photon recycling and luminescent spectral conversion effects [6486-28]

W.-C. Ng, Synopsys, Inc. (USA); G. Letay, Synopsys Switzerland, LLC (Switzerland)

\section{LED MODELING: PHOSPHORS}

$64860 \mathrm{U}$ Statistical analysis and yield management in LED design through TCAD device simulation [6486-29]

G. Létay, Synopsys Switzerland, LLC (Switzerland); W.-C. Ng, Synopsys, Inc. (USA);

L. Schneider, A. Bregy, M. Pfeiffer, Synopsys Switzerland, LLC (Switzerland)

64860V Self-consistent modeling of resonant PL in InGaN SQW LED structure [6486-30]

M. Sabathil, A. Laubsch, N. Linder, Osram Opto Semiconductors (Germany)

64860X A new wafer level coating technique to reduce the color distribution of LEDs [6486-32] B. Braune, K. Petersen, J. Strauss, P. Kromotis, M. Kaempf, Osram Opto Semiconductors (Germany)

64860 Reference-based optical characterization of glass-ceramic converter for high-power white LEDs [6486-33]

A. Engel, M. Letz, T. Zachau, E. Pawlowski, K. Seneschal-Merz, T. Korb, D. Enseling, B. Hoppe, U. Peuchert, Schott AG (Germany); J. S. Hayden, Schott North America (USA)

\section{SESSION 7}

$64860 Z$ Requirements on LEDs for advanced optical systems (Invited Paper) [6486-34]

J. Reill, A. Wilm, Osram Opto Semiconductors (Germany)

648610 Warm white LEDs lighting over $R a=95$ and its applications [6486-35]

K. Kobashi, T. Taguchi, Yamaguchi Univ. (Japan)

648611 Development of light distribution controllable luminaire using high-power LEDs [6486-36] H. Asakawa, Marumo Electric Co., Ltd. (Japan); J. Baba, A. Yaeda, M. Wakaki, Tokai Univ. (Japan) 
648612 A novel temperature compensated operation scheme for trichromatic LED backlights [6486-38]

D.-W. Lee, J.-H. Kim, M.-Y. Park, H.-D. Kim, J.-K. Park, S.-R. Hwang, S.-M. Cho, Samsung

Electro-Mechanics Co., Ltd. (South Korea)

648613 Silicon carbide white light LEDs for solid-state lighting [6486-49]

S. Bet, College of Optics and Photonics, Univ. of Central Florida (USA); N. Quick, Applicote Associates, LLC (USA); A. Kar, College of Optics and Photonics, Univ. of Central Florida (USA)

\section{SESSION 8}

648614 Excitonic effects in ZnO nanowires and hollow nanotubes (Invited Paper) [6486-39] M. Willander, Linköping Univ. (Sweden) and Gothenburg Univ. (Sweden); Y. E. Lozovik, Institute of Spectroscopy (Russia); Q. X. Zhao, O. Nur, Linköping Univ. (Sweden); Q.-H. Hu, P. Klason, Gothenburg Univ. (Sweden)

648616 Fabrication of high-power AllnGaP-based red light emitting diodes with novel package by electroplating [6486-41]

K. C. Chen, Y. K. Su, J. Q. Huang, National Cheng Kung Univ. (Taiwan); C. L. Lin, Kun Shan Univ. (Taiwan); R. W. Chuang, National Cheng Kung Univ. (Taiwan)

648617 Low-resistance and high-reflectivity Aluminum-based reflectors for $p$-GaN flip process [6486-42]

S. W. Chae, K. Y. Ko, D. W. Kim, S. M. Hwang, H. J. Park, J. W. Kim, Y. C. Kim, Samsung

Electro-Mechanics Co., Ltd. (South Korea)

\section{POSTER SESSION}

64861A Developing a new supplemental lighting device with ultra-bright white LED for vegetables [6486-37]

Y. Hu, P. Li, J. Jiang, Jiangsu Univ. (China)

64861B Development of 140-inch autostereoscopic display by use of full-color LED panel [6486-45] H. Nishimura, T. Abe, H. Yamamoto, Y. Hayasaki, N. Nishida, The Univ. of Tokushima (Japan)

64861C High refractive index nanoparticle-loaded encapsulants for light-emitting diodes [6486-48] F. W. Mont, J. K. Kim, M. F. Schubert, H. Luo, E. F. Schubert, R. W. Siegel, Rensselaer Polytechnic Institute (USA)

Author Index 


\title{
Conference Committee
}

\author{
Symposium Chair \\ Yakov Sidorin, Photineer Technology Group (USA) \\ Symposium Cochair
}

Ali Adibi, Georgia Institute of Technology (USA)

Program Track Chair

Daniel K. Johnstone, Virginia Commonwealth University (USA)

Conference Chairs

Klaus P. Streubel, Osram Opto Semiconductors GmbH (Germany)

Heonsu Jeon, Seoul National University (South Korea)

Program Committee

Gerd Bacher, Universität Duisburg-Essen (Germany)

John C. Carrano, Luminex Corporation (USA)

Jonathan J. Halls, Cambridge Display Technology, Ltd. (United Kingdom)

Paul L. Heremans III, IMEC (Belgium)

Michael Heuken, Aixtron AG (Germany)

Masayoshi Koike, Samsung Electro-Mechanics Co., Ltd. (South Korea)

Kurt J. Linden, Spire Corporation (USA)

Heng Liu, AXT, Inc. (USA)

E. Fred Schubert, Rensselaer Polytechnic Institute (USA)

Jerry A. Simmons, Sandia National Laboratories (USA)

Ross P. Stanley, Centre Suisse d'Electronique et de Microtechnique SA

(Switzerland)

Steve A. Stockman, Lumileds Lighting, LLC (USA)

Li-Wei Tu, National Sun Yat-Sen University (Taiwan)

H. Walter Yao, Advanced Micro Devices, Inc. (USA)

John M. Zavada, U.S. Army Research Office (USA)

\section{Session Chairs}

Novel Technologies for LEDs

Klaus P. Streubel, Osram Opto Semiconductors GmbH (Germany)

Fabrication of LEDs and OLEDs

Gerd Bacher, Universität Duisburg-Essen (Germany) 
Organic LEDs

Michael Heuken, Aixtron AG (Germany)

LED Characterization and Reliability

Kurt J. Linden, Spire Corporation (USA)

Photonic Crystals: LED Modeling

Ross P. Stanley, Centre Suisse d'Electronique et de Microtechnique SA (Switzerland)

LED Modeling: Phosphors

Li-Wei Tu, National Sun Yat-Sen University (Taiwan)

Session 7

Heonsu Jeon, Seoul National University (South Korea)

Session 8

Klaus P. Streubel, Osram Opto Semiconductors GmbH (Germany) 